Brit. J. industr. Med., 1960, 17, 125.

$\checkmark$

\title{
ANTISEPTIC CREAM FOR USE ON THE HANDS IN FOOD ESTABLISHMENTS
}

\author{
BY \\ DOREEN L. WEDDERBURN \\ From Unilever Limited, Toilet Preparations Development Unit, Isleworth, Middlesex
}

(RECEIVED FOR PUBLICATION AUGUST 28, 1959)

An antiseptic cream containing benzalkonium chloride as germicide has been developed for use on the hands. It inhibits growth on agar of staphylococci, E. coli, and S. typhimurium; it persists on the fingers, kills organisms applied to the skin, and reduces the normal bacterial population of skin. It is convenient to apply and has been found acceptable by workers in several food factories.

Control of the spread of infection by people handling foodstuffs is directly connected with the general standards of hygiene of operatives and debarring from duty people who are known or suspected carriers of pathogenic infections. In many concerns where food is handled it is not practicable to guard against the employment of carriers because facilities do not exist for testing nasal secretions for pathogenic staphylococci or faeces for intestinal pathogens. Every attempt should therefore be made to minimize the consequences due to transmittable bacteria, e.g., food poisoning and spoiled foods.

Rountree and Thomson (1949) have stated that as many as $60 \%$ of the general population may be nasal carriers of coagulase-positive staphylococci. The carrier rate of pathogenic intestinal bacteria is not nearly as high as this, but obviously the presence of even one such carrier amongst a group of people handling food which will not be cooked before it is eaten constitutes a grave danger to the public. Moss, Squire, Topley, and Johnston (1948) confirmed experimentally that nasal carriers of pathogenic staphylococci also carry the organisms on their hands and showed that transmission by hand is a common occurrence. The spread of pathogenic faecal bacteria by hand is also well known, particularly when adequate standards of personal hygiene are neglected. In the interests of public hygiene and also for the above reasons, people handling food are invariably ordered to wash their hands before beginning work, after lunch and tea breaks, and particularly after visits to the lavatory.

Dipping the hands in a disinfectant solution after washing has several disadvantages, besides being disagreeable and irksome to workers, who, unless supervised, will tend to avoid doing it. Many disinfectants used in this way are odorous and residues left on the hands may contaminate the food, and the solutions frequently cause irritation and superficial drying of the skin. Solutions of quaternary ammonium compounds which are not subject to the above drawbacks have been used, but it has been shown that the initial potency of the quaternary ammonium germicide rapidly becomes reduced by successive hand dipping. This is brought about by the quaternary ammonium compound being taken up on the hands and also by inactivation of the germicide by soap residues left on the hands after washing. Thus the activity of the germicide in a bulk container could be lost in a relatively short time but the fact would remain undetected.

These difficulties can be overcome by the use of an antiseptic cream which is dispensed and used in individual portions. In addition to its antibacterial activity an efficient cream would be expected to be non-greasy, odourless, and to have no harmful effects on the skin.

Benzalkonium chloride is a wide-spectrum quaternary ammonium germicide which has been used in the above-mentioned solutions and is well accepted for skin disinfection in medical practice because of its efficiency and lack of toxicity. Lawrence (1957), in a general review of the quaternary ammonium germicides, mentions the successful use of benzalkonium chloride (also known as "zephiran") both in medical practice and in controlling sanitary conditions in eating and drinking establishments. The toxicology of the compound has been well documented and its use in paediatrics 
recommended by Pfeffer and Smith (1950). Martindale (1958) refers to its use in pre-operative disinfection, obstetrics, and paediatrics, and states that its activity against both Gram-positive and Gram-negative organisms is one and a half times that of "cetrimide". Allport (1952) found that this germicide was less inhibited by the presence of serum and soap than "cetrimide" when using Escherichia coli and Proteus mirabilis as test organisms. For these reasons a cream containing $0.5 \%$ benzalkonium chloride has been developed and tested both in vitro and in vivo. Tests included assessment of efficiency and acceptability under factory conditions and of long-term effects on the skin.

\section{Laboratory Tests}

Many germicides or antiseptics are inactivated to varying extents by the ingredients used to make creams, and preliminary tests were carried out to establish the activity of benzalkonium chloride in various formulae. The cream used for the assessments described in this paper is an unperfumed oil-in-water system made with non-ionic emulsifiers. Care was taken to establish that no incompatibility existed between any of the ingredients and benzalkonium chloride. Non-ionic emulsifiers are known to reduce the activity of many germicides (Wedderburn, 1958) when used at certain concentrations, and for this reason preliminary tests were carried out to select a formula in which the benzalkonium chloride showed maximal activity. When a suitable formula had been found its activity against both Gram-positive and Gram-negative bacteria was assessed. Further tests were then designed to test various aspects of the main requirement of the cream, namely, to prevent transfer of bacteria from the hands of food operatives to the products they handle.

\section{Methods and Results}

(1) Zone Inhibition Test.-The object was to demonstrate the efficiency of benzalkonium chloride in the cream against Micrococcus pyogenes var. aureus N.C.T.C. No. 8244 (coagulase-positive staphylococcus), Escherichia coli (an organism typical of faecal contamination), and Salmonella typhimurium (an organism known to cause food poisoning).

Method.-Nutrient agar plates seeded with the test organisms were prepared. The test cream* $(0 \cdot 1 \mathrm{~g}$.$) and a$ control cream without benzalkonium chloride were placed in separate wells, $5 \mathrm{~mm}$. diameter, cut in the agar. The plates were refrigerated to allow diffusion of the germicide and were then incubated at $37^{\circ} \mathrm{C}$. for 24 hours.

*The antiseptic cream referred to throughout is available from Industrial Soaps Limited.
Results.-The control cream without benzalkonium chloride showed no activity against any of the organisms. The test cream consistently gave a zone of $17 \mathrm{~mm}$. of growth inhibition against Micrococcus pyogenes var. aureus, and zones of $3 \mathrm{~mm}$. and $5 \mathrm{~mm}$. against $E$. coli and $S$. typhimurium respectively.

(2) Fingertip Imprint Tests.-The object was to demonstrate the efficiency of the cream on the living skin against large numbers of Micrococcus pyogenes var. aureus, and to discover whether, when applied to washed hands, the cream would act against extraneous bacteria with which the hands might come into contact. Also the duration of activity under laboratory conditions was assessed.

Method.-Unseeded nutrient agar $(10 \mathrm{ml}$.) was poured into sterile petri dishes. When this had set $10 \mathrm{ml}$. of nutrient agar seeded with $0.05 \mathrm{ml}$. of a 24-hour broth culture of $M$. pyogenes var. aureus was poured on top of the sterile agar to form two-layer plates.

Subjects participating in the test washed their hands with soap and water and rinsed them under running water for five seconds. One gram of the antiseptic hand cream was then applied to the hands and rubbed in. Subjects then carried on with their normal duties which included handling of wet, dry, and greasy objects.

After given time intervals during which the subjects did not wash their hands, the fingertips were placed in contact with the seeded agar for two minutes. The plates were then incubated at $37^{\circ} \mathrm{C}$. for 24 hours.

The assessments were made (a) immediately after application of the cream, (b) half an hour later, $(c)$ one hour later, and $(d)$ two hours later.

At the end of the incubation period the plates were examined for the presence of zones of inhibition in the areas where the fingertips had been in contact. The intensity of growth in and around the fingertip imprint area was recorded as follows:-

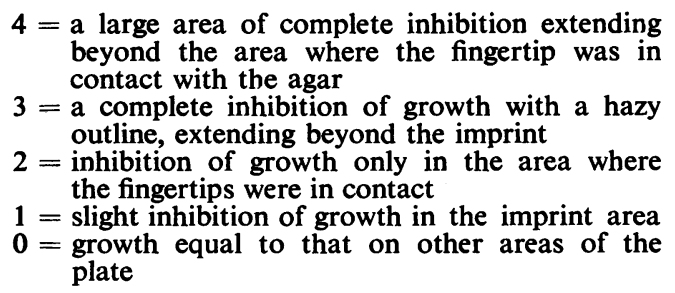

Preliminary experiments had shown that no zone of growth inhibition occurred when fingers covered with a control cream or untreated fingers were laid on the plates, thus any inhibition noted was due to the antiseptic cream.

Results.-A typical result of one such experiment is illustrated in Fig. 1.

This test has been carried out on 21 occasions on subjects doing laboratory work during the two-hour test period, and the results are given in Table 1.

As might be expected there is a very strong association between time and the number of tests with various scores. Table 1 shows that immediately after the antiseptic cream had been applied no subject showed less 


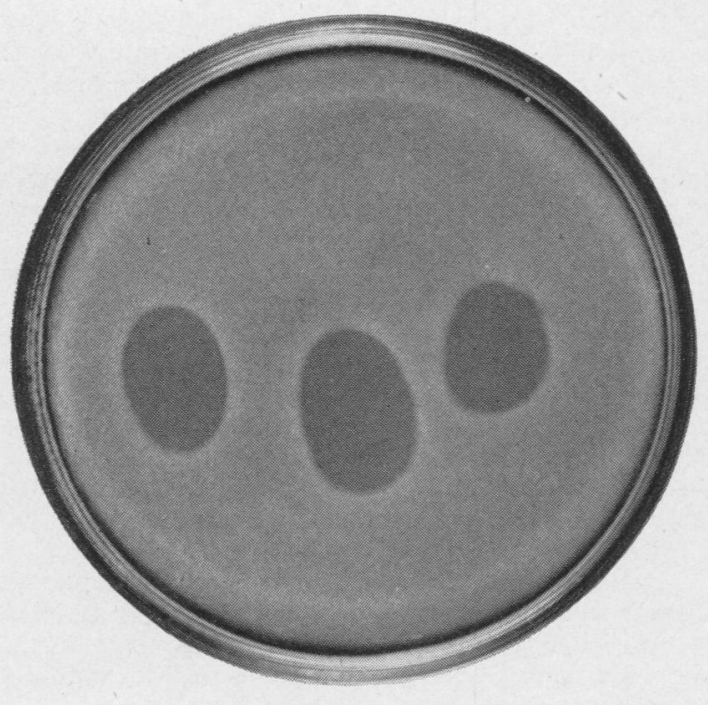

FIG. 1.-The subject laid three fingers on the seeded agar plate one hour after application of the antiseptic cream. This result would be scored 2 (see Table 1).

TABLE 1

RESULTS OF 21 FINGERTIP IMPRINT TESTS*

\begin{tabular}{|c|c|c|c|c|}
\hline \multirow[b]{2}{*}{ Scores } & \multicolumn{4}{|c|}{ No. of Subjects out of 21} \\
\hline & $\begin{array}{l}\text { Immediately } \\
\text { after } \\
\text { Application }\end{array}$ & $\begin{array}{l}\text { Half } \\
\text { Hour } \\
\text { Later }\end{array}$ & $\begin{array}{l}\text { One } \\
\text { Hour } \\
\text { Later }\end{array}$ & $\begin{array}{l}\text { Two } \\
\text { Hours } \\
\text { Later }\end{array}$ \\
\hline $\begin{array}{l}4 \\
3 \\
2 \\
1 \\
0\end{array}$ & $\begin{array}{r}18 \\
3 \\
0 \\
0 \\
0\end{array}$ & $\begin{array}{r}1 \\
16 \\
3 \\
1 \\
0\end{array}$ & $\begin{array}{r}0 \\
3 \\
12 \\
6 \\
0\end{array}$ & $\begin{array}{r}0 \\
0 \\
5 \\
16 \\
0\end{array}$ \\
\hline
\end{tabular}

* The number of subjects recording each of the scores at various time intervals is given. The scores 0 to 4 indicate the degree of inhibition of growth on agar plates as given in the text.

than a score of 3 . Despite a gradual fall in activity, until the majority showed only slight inhibition after two hours, none of the fingertips had entirely lost their activity after this time.

In these tests the occasional presence of organisms other than those seeded in the plates has been noticed in the fingertip imprint area. These were not normal skin residents, but were almost always non-pathogenic spore-bearing organisms probably picked up on the skin during the test period while subjects were carrying out their normal laboratory duties.

(3) Skin Inoculation Test.-This was to demonstrate and assess the effect of the cream on large numbers of bacteria on the skin before application of the cream. The conditions of the test simulated those which would exist if the cream were applied to heavily contaminated skin.

Method.-The inner surfaces of three of the forearms of subjects were washed with soap and water and swabbed with alcohol to remove as many resident bacteria as possible. On each forearm three areas 1 in. $\times 2$ in. were marked with a grease pencil and $0.05 \mathrm{ml}$. of a culture of $M$. pyogenes var. aureus in peptone water was spread evenly over the surface of each rectangle using a sterile glass rod. After the surface was dry (approximately seven minutes) each rectangle was carefully covered with $0.05 \mathrm{~g}$. of cream. On one arm the antiseptic cream was used and on the other arm a cream of the same composition but without the antiseptic. Each area was sampled after 10, 20, and 30 minutes, using an equal number of strokes with swabs made from alginate wool. The swabs were dissolved in a solution of $0.1 \%$ peptone, $1 \%$ "calgon" ( $\mathrm{Na}$ hexametaphosphate), and $4 \%$ Tween 80 . (This concentration of Tween 80 had previously been shown to inactivate fully any benzalkonium chloride carried over on the swabs. This was tested by spreading a thin layer of swab diluent on one half of an agar plate seeded with $M$. pyogenes var. aureus, and assessing inhibition of growth: there was none.) When the swabs had completely dissolved, $1 \mathrm{ml}$. of the solution was added to $100 \mathrm{ml}$. of molten nutrient agar from which five plates were poured. After incubation at $37^{\circ} \mathrm{C}$. for 48 hours the number of colonies was counted.

Results.-This test has been repeated on several occasions with results similar to the typical values given in Table 2.

TABLE 2

RESULT OF SKIN INOCULATION TEST*

\begin{tabular}{|c|c|c|c|c|}
\hline \multirow[b]{2}{*}{ Subject } & \multicolumn{3}{|c|}{$\begin{array}{l}\text { No. of Bacteria } \times 10^{2} \text { on } \\
\text { Each } 2 \text { square inch Area }\end{array}$} & \multirow{2}{*}{$\begin{array}{c}\text { Per- } \\
\text { centage } \\
\text { Reduction } \\
\text { in Mean } \\
\text { Count }\end{array}$} \\
\hline & $\begin{array}{l}\text { Time After } \\
\text { Application of } \\
\text { Cream (min.) }\end{array}$ & $\begin{array}{l}\text { Control } \\
\text { Cream }\end{array}$ & $\begin{array}{c}\text { Antiseptic } \\
\text { Cream }\end{array}$ & \\
\hline 1 & $\begin{array}{l}10 \\
20 \\
30\end{array}$ & $\begin{array}{l}1,746 \\
2,389 \\
1,478\end{array}$ & $\begin{array}{r}156 \\
88 \\
25\end{array}$ & $\begin{array}{l}91 \\
96 \\
98\end{array}$ \\
\hline 2 & $\begin{array}{l}10 \\
20 \\
30\end{array}$ & $\begin{array}{l}13,016 \\
11,691 \\
11,976\end{array}$ & $\begin{array}{l}660 \\
300 \\
272\end{array}$ & $\begin{array}{l}95 \\
98 \\
98\end{array}$ \\
\hline 3 & $\begin{array}{l}10 \\
20 \\
30\end{array}$ & $\begin{array}{r}3,258 \\
12,616 \\
8,458\end{array}$ & $\begin{array}{l}172 \\
101 \\
144\end{array}$ & $\begin{array}{l}94 \\
99 \\
98\end{array}$ \\
\hline All & $\begin{array}{l}10 \\
20 \\
30\end{array}$ & $\begin{array}{l}6,007 \\
8,899 \\
7,284\end{array}$ & $\begin{array}{l}329 \\
101 \\
147\end{array}$ & $\begin{array}{l}94 \cdot 5 \\
98 \\
98\end{array}$ \\
\hline
\end{tabular}

*The skin was covered with a heavy suspension of $M$. pyogenes var, aureus, and, when dry, covered with either the antiseptic or control cream. The number of surviving organisms after 10,20 and 30 minutes was counted, and the reduction under the antiseptic cream compared with that under the control.

The figures show that $91-95 \%$ of the organisms placed on the skin were killed after 10 minutes and the effect tended to increase over a period of half an hour to give up to a $98 \%$ kill. The presence of a quenching agent in the solution in which the swabs were dissolved ensured that the germicide in the antiseptic hand cream had acted while on the skin and that the effect was bactericidal and not bacteriostatic.

In this test a very large number of organisms was applied to the skin in order to exaggerate the probable contamination in practice, and moreover the skin was not 
washed after the organisms were applied and before the creams were used. This situation would hardly prevail in normal circumstances but was adopted to confirm that if, by chance, the cream was applied to a heavily infected skin, a large proportion of the bacteria would be destroyed in situ.

(4) Controlled Tests on Workers' Hands. - These were to assess the activity of the cream on the unwashed hands of subjects carrying out normal laboratory and office duties.

Method.-As in previous experiments a cream without the antiseptic was used as a control. The experiment was carried out on five different days over a period of about two weeks. Groups of approximately 20 subjects who had not washed for at least two hours were used on each day, and $0.2 \mathrm{~g}$. of the antiseptic hand cream was applied by an operator to one hand of each subject, and $0.2 \mathrm{~g}$. of the control cream to the other. On each day half the subjects had the antiseptic hand cream applied to the right hand and the control to the left, while the procedure was reversed for the other half of the subjects. After the cream had been applied the subjects were instructed not to wash their hands for one hour but to carry on with their normal laboratory or office duties. At the end of an hour the hands were swabbed in a controlled manner. Swabs made from alginate wool were used, and samples were taken by stroking the swabs eight times up and down the middle of the back of the hand. Immediately after sampling the swabs were placed in a solution containing $0.1 \%$ peptone, $1 \%$ "calgon", and $4 \%$ Tween 80 as a quenching agent. Duplicate counts made in "roll tubes"* were carried out on the swab diluent.

Results.-In Table 3 each day's results on a group of approximately 20 subjects have been treated as a separate

TABLE 3

RESULTS OF CONTROLLED TESTS ON WORKERS' HANDS*

\begin{tabular}{|c|c|c|c|c|}
\hline \multirow[b]{2}{*}{ Test } & \multirow{2}{*}{$\begin{array}{l}\text { Number of } \\
\text { Subjects }\end{array}$} & \multicolumn{2}{|c|}{ Mean Count per Subject } & \multirow{2}{*}{$\begin{array}{c}\text { Percentage } \\
\text { Reduction } \\
\text { in Mean } \\
\text { Count }\end{array}$} \\
\hline & & $\begin{array}{l}\text { Control } \\
\text { Cream }\end{array}$ & $\begin{array}{c}\text { Antiseptic } \\
\text { Cream }\end{array}$ & \\
\hline $\begin{array}{r}1 \\
2 \\
3 \\
4 \\
5 \\
\text { A11 }\end{array}$ & $\begin{array}{r}21 \\
20 \\
19 \\
22 \\
20 \\
102\end{array}$ & $\begin{array}{r}5,865 \\
2,136 \\
893 \\
1,794 \\
3,037 \\
2,775\end{array}$ & $\begin{array}{r}3,154 \\
675 \\
523 \\
975 \\
2,113 \\
1,504\end{array}$ & $\begin{array}{l}46 \\
68 \\
41 \\
46 \\
30 \\
46\end{array}$ \\
\hline \multicolumn{4}{|c|}{$95 \%$ confidence limits for mean } & $63 \cdot 4-29 \cdot 0$ \\
\hline
\end{tabular}

*The number of viable bacteria on the backs of unwashed hands treated with either the control or antiseptic cream was counted. The figures given are averages for each of the five occasions on which The figures given are averages for each of the five occasions on which
the test was carried out together with an overall average for 102 tests on each cream.

test. It will be seen that the antiseptic cream reduced the bacterial flora of the hands by an average of $46 \%$.

This test gave a smaller percentage reduction in mean count than previous experiments. This is probably due in part to the great variation in the number of organisms

*Astell Roll Tubes, Lab. Pract., 6, No. 11, 635 (1957). on the skin. This variation occurs not only from subject to subject and from day to day but also from the left to the right hand and even from one part of the hand to another.

Preliminary experiments had revealed the existence of these variations. The 102 comparative counts taken from hands treated with the antiseptic or control cream were therefore submitted to statistical analysis.

Significance tests were carried out to determine whether the difference in numbers of bacteria on hands treated with the antiseptic cream and the control depended on $(a)$ the subject, $(b)$ the occasion, $(c)$ whether the left or right hand was treated with the antiseptic cream.

The tests showed that the difference did not depend on any of these, and that by pooling all the results the effect of the antiseptic cream was significant at the 0.05 level.

Thus the antiseptic cream gave a considerable reduction although this was not as great as in the skin inoculation test where the numbers of bacteria on the skin were constant and the conditions of test rigorously controlled.

(5) Dermal Toxicity Test.-This was to determine whether frequent application of the cream over a period of several weeks would cause irritation of, or have any observable effect on, the skin.

Method and Result.-Fifteen subjects applied the antiseptic hand cream between 10 and 14 times a day for a period of three weeks. Some of the subjects were known to have sensitive skins, which became dry, particularly in cold weather, but none showed any sign of skin irritation or other effect during this somewhat exaggerated experiment.

\section{Factory Test}

Uncontrolled User Test.-This was to assess the acceptance of the cream by factory staff and also to determine its efficiency in use.

Method.-The test was carried out in a food factory and dispensers were set up at five different washing points. Two dispensers were employed at each washing point, one of which contained the antiseptic hand cream and the other a standard barrier cream. The test subjects were instructed to use either the barrier cream or the antiseptic hand cream after washing. The appropriate cream was to be rubbed into the hands at the start of work, after lunch and tea breaks, and after using the lavatory. The tests were carried out over a period of six weeks and each day samples were taken from some of the subjects using either the antiseptic cream or the barrier cream.

The samples were examined, by plating on MacConkey agar, for the presence of coliform organisms which are accepted as indicators of faecal contamination. In all, 90 samples were taken, 45 from subjects using the antiseptic hand cream and 45 from those using the barrier cream.

Results.-Coliform organisms were isolated from seven out of 45 samples taken from subjects using the 
barrier cream. On no occasion were coliforms discovered in any of the $\mathbf{4 5}$ samples from subjects using the antiseptic cream. This degree of contrast is sufficient to indicate with a $95 \%$ probability that the difference between the two creams is real.

\section{Discussion and Conclusions}

There is little doubt that the hands of food operatives are a source of pathogenic contamination and that in establishments where food which will not be cooked before it is eaten, or where food wrappings are handled, every precaution should be taken to minimize the risks. Facilities for frequent hand washing should be provided and the use of nail brushes encouraged. Where possible people with cuts, sores, or scratches on their hands should be debarred from duty, and in instances where the use of handling tongs or rubber gloves is considered desirable, an antiseptic hand cream should not be thought of as a substitute.

The main functions of an antiseptic cream are to reduce the numbers of viable bacteria left on the skin after washing and to provide an invisible, odourless film on the hands which will prevent the transfer of pathogenic organisms. It is an adjunct to and not a substitute for normal hygiene routines, and may help to reduce chapping which is frequently accompanied by superficial infection.

The tests carried out on the cream under test gave the following results:-

(1) It was active against pathogenic organisms likely to be met with in practice, such as $M$. pyogenes var. aureus and $S$. typhimurium.

(2) The inhibitory film produced by applying the cream to the fingertips remained active for at least two hours during which both wet and dry work was carried out.

(3) When applied to skin deliberately contaminated with large numbers of bacteria, the cream was bactericidal to over $90 \%$ of the organisms after 10 minutes, and after 30 minutes up to $98 \%$ of the organisms had been destroyed.

The bactericidal as distinct from bacteriostatic properties of the cream were demonstrated (in Experiment 3) by the presence of an inactivator in the swab diluent. This showed that the cream acted while on the skin, and not after both organisms and cream had been removed by swabbing.

(4) In practical trials, in which the cream was applied to unwashed skin bearing its normal flora and subjects performed a variety of office and laboratory tasks during the test period, the cream considerably reduced the bacterial population on the hands when compared with a control cream without benzalkonium chloride.

(5) Dermal toxicity tests designed to reveal skin irritation showed that frequent application of the cream caused no adverse effects. In fact, such applications improved the skin condition of most of the 15 subjects. The number of subjects was too small to assess the potential sensitizing properties of the cream, but as no reference to skin sensitization by benzalkonium chloride could be found in the literature, and as over 500 people have used this cream for periods of over a year without adverse effect, the likelihood of sensitization is small.

(6) Actual user tests in a food factory showed that contamination by faecal organisms was eliminated when the antiseptic cream was used, whereas a barrier cream without benzalkonium chloride permitted the survival of $E$. coli on the hands of several workers.

The activity of the cream is destroyed by washing the hands with soap and water but this is not considered a disadvantage as the cream is intended for use immediately after hand washing and not as a substitute. For fullest activity, hands should be thoroughly rinsed after soaping and before applying the cream, because of the known incompatibility between soap and benzalkonium chloride. Traces of soap left on the hands do not, however, inactivate the cream significantly, owing to the excess of benzalkonium chloride deposited on the skin when the cream is applied. The cream has been well accepted by operatives in large food factories during a period of one to two years.

I am grateful to Unilever Limited for permission to publish this work and to the managements of the ice cream and meat and handy food factories of $\mathrm{T}$. Wall and Sons Limited where the trials were carried out. I also thank Mr. E. Green for valuable assistance in the statistical interpretation of the results and Mrs. S. F. Dunbar, Mrs. J. Branson, and Miss P. Brooks for their technical assistance.

\section{REFERENCES}

Allport, N. L. (1952). Pharm. J., 169, 435.

Lawrence, C. A. (1957). In Antiseptics, Disinfectants, Fungicides, and Chemical and Physical Sterilization. Ed. Reddish, G. F., 2nd ed., p. 581. Henry Kimpton, London.

Martindale (1958). The Extra Pharmacopoeia, 24th ed., Vol. 1, p. 366. Pharmaceutical Press, London.

Moss, B., Squire, J. R., Topley, E., and Johnston, C. M. (1948). Lancet, 1, 320.

Pfeffer, W., and Smith, C. A. (1950). J. Pediat., 37, 351.

Rountree, P. M., and Thomson, E. F. (1949). Lancet, 2, 501.

Wedderburn, D. L. (1958). J. Soc. cosmet. Chem., 9, 210. 